University of Wollongong

Research Online

Australian Institute for Innovative Materials -

Papers

Australian Institute for Innovative Materials

$1-1-2014$

\title{
SOC estimation for LiFePO4 battery in EVs using recursive least-squares with multiple adaptive forgetting factors
}

\author{
Van Huan Duong \\ University of Wollongong, vhd931@uowmail.edu.au \\ Hany A. Bastawrous \\ University of Wollongong, hany@uow.edu.au \\ Kai Chin Lim \\ University of Wollongong, kcl502@uowmail.edu.au \\ Khay Wai W. See \\ University of Wollongong, kwsee@uow.edu.au \\ Peng Zhang \\ University of Wollongong, pengz@uow.edu.au
}

See next page for additional authors

Follow this and additional works at: https://ro.uow.edu.au/aiimpapers

Part of the Engineering Commons, and the Physical Sciences and Mathematics Commons

Research Online is the open access institutional repository for the University of Wollongong. For further information contact the UOW Library: research-pubs@uow.edu.au 


\title{
SOC estimation for LiFePO4 battery in EVs using recursive least-squares with multiple adaptive forgetting factors
}

\author{
Abstract \\ This work presents a novel technique which is simple yet effective in estimating electric model \\ parameters and state-of-charge (SOC) of the LiFePO4 battery. Unlike the well-known recursive least- \\ squares-based algorithms with single constant forgetting factor, this technique employs multiple adaptive \\ forgetting factors to provide the capability to capture the different dynamics of model parameters. The \\ validity of the proposed method is verified through experiments using actual driving cycles.

\section{Keywords} \\ estimation, lifepo4, battery, evs, recursive, least, soc, squares, factors, multiple, adaptive, forgetting \\ Disciplines \\ Engineering | Physical Sciences and Mathematics

\section{Publication Details} \\ Duong, V. H., Bastawrous, H. A., Lim, K. C., See, K. W., Zhang, P. \& Dou, S. X. (2014). SOC estimation for \\ LiFePO4 battery in EVs using recursive least-squares with multiple adaptive forgetting factors. Connected \\ Vehicles and Expo (ICCVE), 2014 International Conference on (pp. 520-521). United States: Institute of \\ Electrical and Electronics Engineers.

\section{Authors} \\ Van Huan Duong, Hany A. Bastawrous, Kai Chin Lim, Khay Wai W. See, Peng Zhang, and S X. Dou
}




\title{
SOC Estimation for $\mathrm{LiFePO}_{4}$ Battery in EVs Using Recursive Least-Squares with Multiple Adaptive Forgetting Factors
}

\author{
V. H. Duong, Student Member, IEEE, H. A. Bastawrous, Member, IEEE, \\ K. C. Lim, Student Member, IEEE, K. W. See, P. Zhang, and S. X. Dou \\ Institute for Superconducting and Electronic Materials, University of Wollongong, NSW, Australia
}

\begin{abstract}
This work presents a novel technique which is simple yet effective in estimating electric model parameters and state-of-charge (SOC) of the $\mathrm{LiFePO}_{4}$ battery. Unlike the wellknown recursive least-squares-based algorithms with single constant forgetting factor, this technique employs multiple adaptive forgetting factors to provide the capability to capture the different dynamics of model parameters. The validity of the proposed method is verified through experiments using actual driving cycles.
\end{abstract}

Keywords- State-of-Charge, $\mathrm{LiFePO}_{4}$ Battery, Recursive Least Square, Multiple Adaptive Forgetting Factors

\section{INTRODUCTION}

Electric vehicles have been well recognized because of their contribution in the promising future of emission-free transportation means. The core of the electric vehicles is the battery storage system which plays an important role in the safety and price of the vehicles. Therefore, there is a necessity to develop an effective battery management system in the field of electrification. Various advanced methods have been proposed and applied to cope with difficulties in estimating the states of battery in the storage system [1].

Among these effective methods, recursive least-squares (RLS) algorithm applied on electrical-equivalent battery model has been well proposed and implemented in the battery management system in order to estimate the states of the $\mathrm{LiFePO}_{4}$ battery online $[1,2]$. In order to adapt with dynamic changes of the battery under driving cycles, RLS with a constant forgetting factor has been used. However, the parameters to be estimated for the battery model have different dynamic characteristics. Hence, assigning a single constant forgetting factor may not provide accurate estimations of all battery model parameters and will likely cause divergence in the estimation. To further improve the performance of RLS algorithm, a novel technique employing multiple adaptive forgetting factors is proposed for the first time in this paper.

\section{BATtery Model AND Estimation AlgORithm}

\section{A. Battery Model}

In order to accurately model the $\mathrm{LiFePO}_{4}$ battery, an equivalent circuit consisting of an open-circuit voltage (OCV) source, an internal resistance, and two networks of resistance and capacitance (R-RC-RC) connected in series is required [3]. However, this model requires heavy computations due to

This work is supported by AutoCRC Ltd of Australia, Malaysian Automotive Institute, and Australian Institute of Innovative Materials, UOW

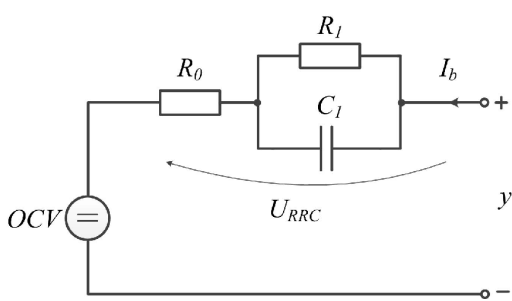

Fig. 1 Electrical model for $\mathrm{LiFePO}_{4}$ battery

the presence of two capacitors. To simplify the model for the sake of fast and feasible implementation, it can be transformed into the one which is depicted in Fig. 1. A detailed explanation of the validity and effectiveness of this transformation can be found elsewhere [4].

\section{B. Estimation Algorithm}

In order to apply the proposed RLS technique, an auto regressive exogenous (ARX) model is required. To do so, firstly the transfer function of the battery impedance is expressed in s-domain as follows:

$$
G(s)=\frac{U_{R R C}(s)}{I(s)}=R_{0}+\frac{R_{1}}{1+s R_{1} C_{1}}
$$

where $U_{R R C}$ represents the dynamic voltage drop of the battery across the series resistance and the RC section. Then, the system is discretised with a sampling time $T_{s}$ through the simple forward-difference transformation to avoid complexity. Finally, the ARX model is formulated as below:

$$
\begin{aligned}
y_{k} & =\boldsymbol{\theta}_{k}^{T} \cdot \boldsymbol{\phi}_{k} \\
\text { where } & \boldsymbol{\theta}_{k}=\left[\begin{array}{lll}
b_{0, k} ; & b_{1, k} ; & a_{1, k} ; \quad O C V_{k}
\end{array}\right] \\
\boldsymbol{\phi}_{k} & =\left[\begin{array}{lll}
I_{k} ; & I_{k-1} ; \quad\left(O C V_{k-1}-y_{k-1}\right) ; \quad 1
\end{array}\right] \\
b_{0} & =R_{0} \\
b_{1} & =-R_{0}+\frac{T_{s}}{C_{1}}+\frac{T_{s} \cdot R_{0}}{R_{1} \cdot C_{1}} \\
a_{1} & =\frac{T_{s}}{R_{1} \cdot C_{1}}-1
\end{aligned}
$$

For the conventional RLS, usually a single forgetting factor $\lambda$ is assigned for all the parameters although the dynamic behaviour of each parameter is different from the others under the same conditions of current, voltage, and temperature of the battery. This might result in an inaccurate parameters identification which consequently causes a poor SOC estimation. To avoid this issue, we propose to employ 


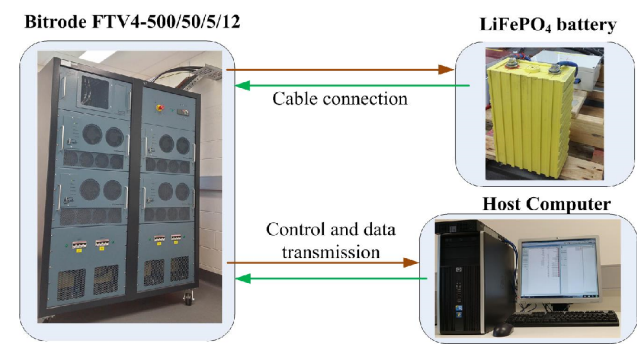

Fig. 2 Test bench configuration

multiple adaptive forgetting factors $\lambda_{i, k}$ in the computation procedure of the RLS estimation as follows $[5,6]$ :

$$
\begin{aligned}
& \lambda_{i, k}=1-\frac{1}{1+\zeta_{i} / \phi_{i, k}^{T} \cdot P_{i, k-1} \cdot \phi_{i, k}} \\
& L_{i, k}=\frac{P_{i, k-1} \cdot \phi_{i, k}}{\lambda_{i, k}+\phi_{i, k}^{T} \cdot P_{i, k-1} \cdot \phi_{i, k}} \\
& P_{i, k}=1 / \lambda_{i, k}\left(1-L_{i, k} \cdot \phi_{i, k}^{T}\right) P_{i, k-1} \\
& \boldsymbol{\theta}_{k}=\boldsymbol{\theta}_{k-1}+\boldsymbol{L}_{m u l, k}\left(y_{k}-\boldsymbol{\phi}_{k}^{T} \cdot \boldsymbol{\theta}_{k-1}\right)
\end{aligned}
$$

where $\quad \boldsymbol{L}_{m u l, k}=\frac{1}{1+\sum_{i=1}^{4} \frac{P_{i, k-1} \cdot \phi_{i, k}^{2}}{\lambda_{i, k}}}\left[\begin{array}{c}P_{1, k-1} \cdot \phi_{1, k} / \lambda_{1, k} \\ P_{2, k-1} \cdot \phi_{2, k} / \lambda_{2, k} \\ P_{3, k-1} \cdot \phi_{3, k} / \lambda_{3, k} \\ P_{4, k-1} \cdot \phi_{4, k} / \lambda_{4, k}\end{array}\right]$

$\boldsymbol{L}_{m u l, k}$ and $L_{i, k}$ are the updated gain of estimated parameters vector $\boldsymbol{\theta}_{k}$ and its component, respectively. Similarly, $\lambda_{i}$ and $P_{i, k}$ are the forgetting factor and the covariance error of each component of vector $\boldsymbol{\theta}_{k}$ while $\zeta_{i}$ is the tunning parameter for each forgetting factor.

\section{EXPERIMENTAL RESULTS}

A $40 \mathrm{Ah} \mathrm{LiFePO}$ battery is used for the experiments which are carried out at $20^{\circ} \mathrm{C}$. The test bench configuration is illustrated in Fig. 2. A programmable Bitrode machine with very high accuracy is used to charge/discharge the battery with maximum current of 500A and maximum voltage of $12 \mathrm{~V}$. Current pulses are applied to construct the battery's characteristic curve of OCV versus SOC. Then, experiments using current patterns and battery terminal voltage of actual driving cycle are conducted to verify the validity of the proposed technique as shown in Fig. 3. The battery parameters are directly identified based on the estimation of the parameters vector $\boldsymbol{\theta}_{k}$. The SOC is obtained from the estimated OCV via a look-up table built from the experimental OCVSOC curve. It can be seen in Fig. 4 (a) that estimation of the resistance $R_{0}$ is constant after convergence which perfectly describes the limited conductance of the contact, the inter-cell connections, and the electrolyte. In Fig. 4 (b), the estimation of the charge-transfer resistance $R_{l}$ is dynamic corresponding

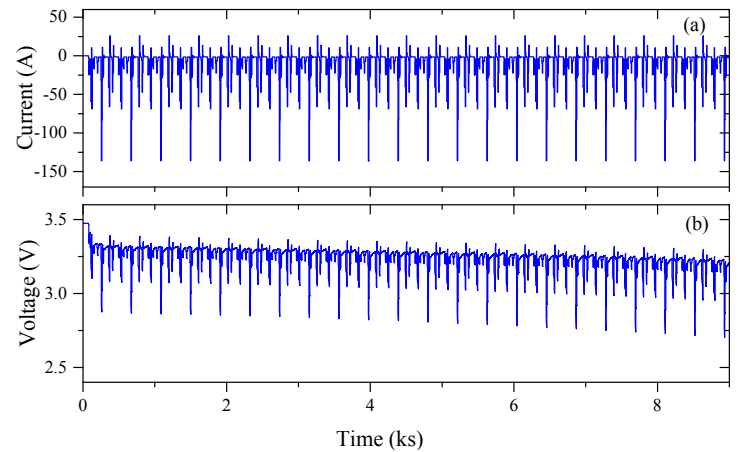

Fig. 3 Actual driving cycle load profile: (a) Current, (b) Voltage

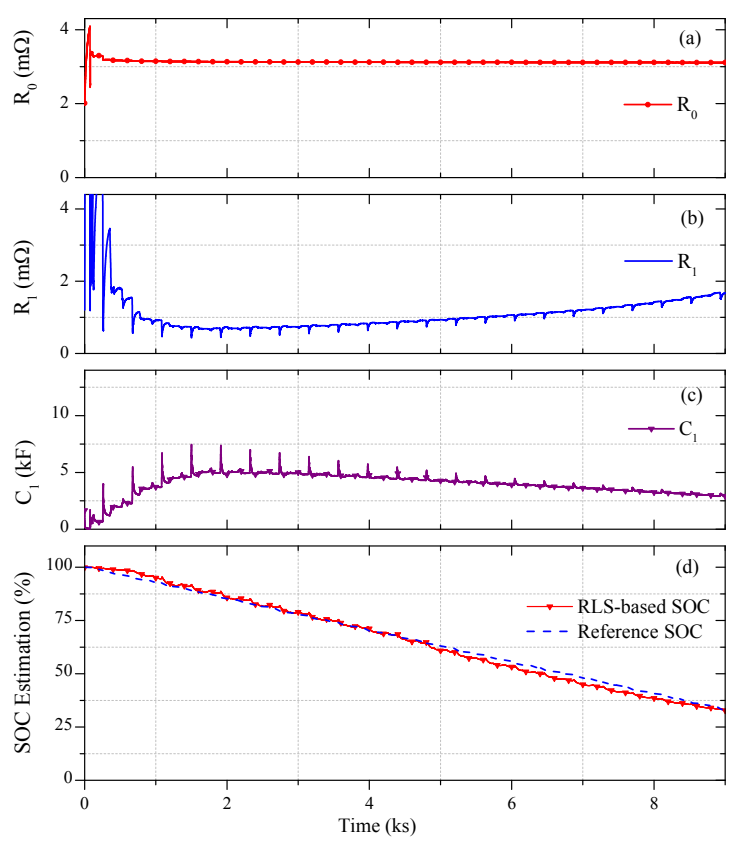

Fig. 4 Experimental results: (a-c) parameters estimation; (d) SOC estimation

to the change of the current amplitude and SOC as expected. Fig. 4 (d) shows the result of SOC estimation obtained by the proposed method compared to the reference SOC obtained from Coulomb counting with an accurate initial value. As can be seen, the estimated SOC tracks the reference value well with root-mean-square error (RMSE) of 0.019 .

\section{CONCLUSION}

In this work, the RLS technique with multiple adaptive forgetting factors has been proposed for the estimation of the dynamic parameters and $\mathrm{SOC}$ of $\mathrm{LiFePO}_{4}$ battery. The validity of the method has been confirmed by accurate SOC estimation results with RMSE of 0.019 . In addition, the feasibility of this method has been proven by the simplicity of the model and the light scalar computations in algorithm.

\section{REFERENCES}

[1] W. Waag, et al., Journal of Power Sources, vol. 258, pp. 321-339, 2014.

[2] H. He, et al.,Energy, vol. 39, pp. 310-318, 2012.

[3] L. Long, et al., INTELEC IEEE 33rd International, pp. 1-9, 2011.

[4] V.-H. Duong, et al., The Trans. of the KIPE, vol. 19, pp. 139-146, 2014

[5] D. C. Huynh, et al., Power and Energy IEEE conf., pp. 444-449, 2010.

[6] A. Vahidi, et al., Vehicle System Dynamics, vol. 43, pp. 31-55, 2005. 